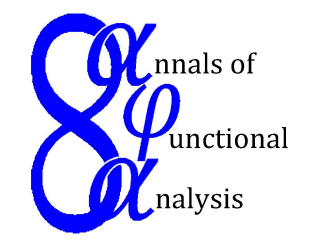

Ann. Funct. Anal. 5 (2014), no. 1, 128-142

$\mathscr{A}$ NNALS OF $\mathscr{F}$ UNCTIONAL $\mathscr{A}$ NALYSIS

ISSN: 2008-8752 (electronic)

URL:www.emis.de/journals/AFA/

\title{
A SETTING FOR GENERALIZED ORTHOGONALIZATION
}

\author{
GUSTAVO CORACH* AND ALEJANDRA MAESTRIPIERI
}

This paper is dedicated to Professor Tsuyoshi Ando, with affection and admiration

Communicated by L. Székelyhidi

\begin{abstract}
Let $\mathcal{H}$ be a complex Hilbert space. We study the geometry of the space of pairs $(A, E)$, for $A$ a (semidefinite bounded linear) positive operator on $\mathcal{H}$ and $E$ a (bounded linear) projection on $\mathcal{H}$ such that $A E=E^{*} A$.
\end{abstract}

\section{INTRODUCTION}

An orthogonalization method in a Hilbert space $\mathcal{H}$ is a tool which converts a sequence of linearly independent vectors in a sequence of orthonormal vectors, with some additional properties. But one can also think that an orthogonalization method provides to every closed subspace $\mathcal{S}$ of $\mathcal{H}$ a projection with image $\mathcal{S}$ which is orthogonal with respect to a certain inner product. In this sense, a setting which unifies different orthogonalization methods is given by the set $\mathcal{Z}$ consisting of all pairs $\left(\langle,\rangle^{\prime}, E\right)$, where $\langle,\rangle^{\prime}$ is a inner product or, more generally, a semi-inner product on $\mathcal{H}$ and $E$ is a bounded linear projection acting on $\mathcal{H}$. In order to maintain the situation under control, we shall only admit bounded semi-inner products (with respect to the original inner product $\langle$,$\rangle of \mathcal{H}$ ). Therefore, $\langle,\rangle^{\prime}$ is indeed determined by a positive (semidefinite bounded linear) operator $A$ acting on $\mathcal{H}$, by the rule $\langle\xi, \eta\rangle^{\prime}=\langle A \xi, \eta\rangle$, where $\xi, \eta \in \mathcal{H}$. Thus, the object of our present study is the set

$$
\mathcal{Z}=\left\{(A, E) \in L(\mathcal{H})^{+} \times \mathcal{Q}: A E=E^{*} A\right\}
$$

where $L(\mathcal{H})^{+}$is the cone of positive operators and $\mathcal{Q}$ is the subset of all (bounded linear) projections acting on $\mathcal{H}$. The identity $A E=E^{*} A$ says, exactly, that the

Date: Received: 1 July 2013; Accepted: 18 September 2013.

* Corresponding author.

2010 Mathematics Subject Classification. Primary 46C50; Secondary 46C05, 47A62.

Key words and phrases. A-selfadjoint projections, positive operators, orthogonal projections. 
projection $E$ is Hermitian with respect to the semi-inner product $\langle,\rangle^{\prime}$ determined by $A$.

For a fixed Hilbert space $\mathcal{H}$, we say that a closed subspace $\mathcal{S}$ is compatible with a positive (semidefinite bounded linear) operator $A$ if there exists a (bounded linear) projection $E$ on $\mathcal{H}$ with image $\mathcal{S}$ such that $A E=E^{*} A$. Thus, such $E$ behaves like an orthogonal projection onto $\mathcal{S}$ with respect to the semi-inner product $\langle,\rangle_{A}$. Compatibility is automatic if $\mathcal{H}$ has finite dimension. However, in the infinite dimensional case, there exist pairs $(A, \mathcal{S})$ such that no such $E$ exists. If

$$
\mathcal{P}(A, \mathcal{S})=\left\{E \in L(\mathcal{H}): E^{2}=E, \mathcal{R}(E)=\mathcal{S}, A E=E^{*} A\right\}
$$

then $(A, \mathcal{S})$ is compatible if $\mathcal{P}(A, \mathcal{S})$ is not empty. If $A$ is invertible then $(A, \mathcal{S})$ is compatible for every $\mathcal{S}$ and $\mathcal{P}(A, \mathcal{S})$ contains a single projection, denoted by $P_{A, \mathcal{S}}$. The notion of compatibility has been implicitly used by A. Sard [21] in 1950' and later by Hassi and Nordström [12], for $A$ Hermitian. In [1], Ando defined the notion of complementable matrices by a subspace, as a generalization of Schur complements. It turns out that compatibility and complementability for selfadjoint operators on a Hilbert space are equivalent, see [6]. For a complete discussion on compatibility matters amd applications, the reader is referred to the papers [5], [6], [7], [8], [16], [17]; see also [18].

Some relevant results are obtain if $A \in G L(\mathcal{H})^{+}$of all positive invertible operators. In particular, many facts are proven not for $\mathcal{Z}$ but for the set

$$
\mathcal{Z}^{\circ}=\left\{(A, E) \in \mathcal{Z}: A \in G L(\mathcal{H})^{+}\right\} .
$$

In some sense, the main interest of the sets $\mathcal{Z}$ and $\mathcal{Z}^{\circ}$ is that every oblique projection $E \in \mathcal{Q}$ is paired with all the semi-inner products $\langle,\rangle_{A}$ under which $E$ is orthogonal. This offers and interesting setting for problems where perturbations of the scalar product appear naturally. The reader is referred to the paper by Pasternak-Winiarski [19] with relevant bibliography. Also, in problems where the conjugate gradient method is used (see, e.g., [13]) a setting like the mentioned above may be useful.

The contents of the paper are the following. In Section 2 we collect some notation and known facts about Dixmier angles between closed subspaces of a Hilbert space. We also survey results about compatibility, referring the reader to the papers [5], [6], [7], [8], [16], [17] for the corresponding proofs. However, we include with a proof, a new result on compatibility, namely, that for a fixed $A \in L(\mathcal{H})^{+}$every closed subspace $\mathcal{S}$ of $\mathcal{H}$ is compatible with $A$ if and only if $\mathcal{R}(A)$ is closed and $\operatorname{dim} \mathcal{R}(A)<\infty$ or $\operatorname{dim} \mathcal{N}(A)<\infty$, where $\mathcal{R}(A)$ denotes the image of $A$ and $\mathcal{N}(A)$ denotes its nullspace. In Section 3 we describe in several ways the set $\mathcal{Z}$ and its natural projections onto $L(\mathcal{H})^{+}$and $\mathcal{Q}$. The fibers $\mathcal{Z}_{A}$ of the first projection are described in Section 4. Section 5 contains a similar study for the fibers $\mathcal{Z}^{E}$ of the second projection. However, it should be noticed that the similarity is only formal. In fact, each $\mathcal{Z}^{E}$ is convex and, so topologically quite simple. On the other side, each $\mathcal{Z}_{A}$ is as complex as the set $\mathcal{Q}$. Both sections 4 and 5 contain result describing some intersections of the type $\mathcal{Z}_{A_{1}} \cap \mathcal{Z}_{A_{2}}, \mathcal{Z}^{E_{1}} \cap \mathcal{Z}^{E_{2}}$, respectively. Finally, at Section 6 we study some topological facts about $\mathcal{Z}$ and $\mathcal{Z}^{\circ}$. In particular, we determine the connected components of $\mathcal{Z}$ and $\mathcal{Z}^{\circ}$. 


\section{Compatible pairs}

Let $G r=G r(\mathcal{H})$ denote the Grassmann manifold of $\mathcal{H}$, i.e., the set of all closed subspaces $\mathcal{S}$ of $\mathcal{H}$. Let $L(\mathcal{H})$ denote the algebra of linear bounded operators acting on $\mathcal{H}$ and for $T \in L(\mathcal{H})$, denote by $\mathcal{R}(T)$ the range of $T$ and by $\mathcal{N}(T)$ its nullspace. Given $A \in L(\mathcal{H})^{+}$and $\mathcal{S} \in G r$ we say that they are compatible (or that the pair $(A, \mathcal{S})$ is compatible) if there exists $E \in \mathcal{Q}$ such that $A E=E^{*} A$ and $\mathcal{R}(E)=\mathcal{S}$. As we said in the introduction, this means that $E$ is Hermitian with respect to the semi inner product $\langle,\rangle_{A}$ defined as $\langle x, y\rangle_{A}:=\langle A x, y\rangle, \forall x, y \in \mathcal{H}$. We refer the reader to the papers [5], [6], [8] for the proofs of the results mentioned below. Theorem 2.1 is new and we present a proof. The pair $(A, \mathcal{S})$ is compatible if and only if $\mathcal{S}+(A \mathcal{S})^{\perp}=\mathcal{H}$. Now, if we defined the Dixmier angle between two closed subspaces $\mathcal{M}, \mathcal{W}$ as the unique $\alpha \in[0, \pi / 2]$ such that

$$
\cos \alpha=c_{0}(\mathcal{M}, \mathcal{W})=\sup \{|\langle m, w\rangle|: m \in \mathcal{M}, w \in \mathcal{W},\|m\|=\|w\|=1\},
$$

then it follows that $(A, \mathcal{S})$ is compatible if and only if $c_{0}\left(\mathcal{S}^{\perp}, \overline{A \mathcal{S}}\right)<1$. If $A \in$ $G L(\mathcal{H})^{+}$then $(A, \mathcal{S})$ is compatible for every $\mathcal{S} \in G r$. In fact in this case $\langle,\rangle_{A}$ is equivalent to the original $\langle$,$\rangle and then, \mathcal{H}_{A}=\left(\mathcal{H},\langle,\rangle_{A}\right)$ is also a Hilbert space, so that for every closed subspace $\mathcal{S}$ (of $\mathcal{H}$ or, indistinctly, $\mathcal{H}_{A}$ ) there exists a unique $A$-orthogonal projection $P_{A, \mathcal{S}}$ onto $\mathcal{S}$. It easily follows that $A P_{A, \mathcal{S}}=P_{A, \mathcal{S}}^{*} A$.

Concerning the existence of pairs $(A, \mathcal{S})$ which are not compatible, one can show some examples (see, for instance, [5]). However, the following result gives a complete answer.

Theorem 2.1. Let $A \in L(\mathcal{H})^{+}$. Then $(A, \mathcal{S})$ is compatible for every $\mathcal{S} \in G r$ if and only if $\mathcal{R}(A)$ is closed and $\operatorname{dim} \mathcal{R}(A)<\infty$ or $\operatorname{dim} \mathcal{N}(A)<\infty$.

Proof. Suppose that $\mathcal{R}(A)$ is closed and consider $\mathcal{S} \in G$ r. If $\operatorname{dim} \mathcal{R}(A)<\infty$ then every subset of $\mathcal{R}(A)$ has finite dimension and therefore is closed. Then $c_{0}\left(\overline{A \mathcal{S}}, \mathcal{S}^{\perp}\right)=c_{0}\left(A \mathcal{S}, \mathcal{S}^{\perp}\right)<1$, because $A \mathcal{S} \cap \mathcal{S}^{\perp}=\{0\}$ and $A \mathcal{S}$ has finite dimension. If $\operatorname{dim} \mathcal{N}(A)<\infty$ then $\mathcal{S}+\mathcal{N}(A)$ is automatically closed; this condition, if $A$ has closed range, is equivalent to the compatibility of $A$ and $\mathcal{S}$, see [5].

Conversely, suppose that $\mathcal{R}(A)$ is not closed; consider $y \in \overline{\mathcal{R}(A)}$ such that $y \notin \mathcal{R}(A), y \neq 0$ and consider $\mathcal{S}^{\perp}=[y]$ the subspace generated by $y$. Then $(A \mathcal{S})^{\perp}=A^{-1}\left(\mathcal{S}^{\perp}\right)=A^{-1}([y] \cap \mathcal{R}(A))=A^{-1}(\{0\})=\mathcal{N}(A)$. Therefore, by taking orthogonal complement, $\overline{A \mathcal{S}}=\overline{\mathcal{R}(A)}$; in this case $\overline{A \mathcal{S}} \cap \mathcal{S}^{\perp}=\overline{\mathcal{R}(A)} \cap \mathcal{S}^{\perp}=\mathcal{S}^{\perp}=$ $[y] \neq\{0\}$. Hence $c_{0}\left(\overline{A \mathcal{S}}, \mathcal{S}^{\perp}\right)=1$ so that $(A, \mathcal{S})$ is not compatible. See [2].

If $\mathcal{R}(A)$ is closed and $\operatorname{dim} \mathcal{R}(A)=\operatorname{dim} \mathcal{N}(A)=\infty$, then it is possible to construct a closed subspace $\mathcal{S}$ such that $c(\mathcal{S}, \mathcal{N}(A))=1$, or equivalently, such that $\mathcal{S}+\mathcal{N}(A)$ is not closed, see [11]. Therefore $(A, \mathcal{S})$ is not compatible. This ends the proof.

If $(A, \mathcal{S})$ is compatible, then the set

$$
\mathcal{P}(A, \mathcal{S})=\left\{E \in \mathcal{Q}: \mathcal{R}(E)=\mathcal{S}, A E=E^{*} A\right\}
$$

is not empty. It is a closed affine manifold of $L(\mathcal{H})$, with a distinguished element $P_{A, \mathcal{S}}$ which corresponds to the direct decomposition $\mathcal{H}=\mathcal{S} \dot{+}\left((A \mathcal{S})^{\perp} \ominus \mathcal{S}\right)$, where the sum is direct and $(A \mathcal{S})^{\perp} \ominus \mathcal{S}:=(A \mathcal{S})^{\perp} \cap\left(\mathcal{S} \cap(A \mathcal{S})^{\perp}\right)^{\perp}$. It can be shown 
that $\mathcal{P}(A, \mathcal{S})$ contains a single element if and only if $(A, \mathcal{S})$ is compatible and $\mathcal{N}(A) \cap \mathcal{S}=\{0\}$. In fact $\mathcal{P}(A, \mathcal{S})$ can be parametrized as

$$
\begin{aligned}
\mathcal{P}(A, \mathcal{S})= & P_{A, \mathcal{S}}+L\left(\mathcal{S}^{\perp}, \mathcal{S} \cap \mathcal{N}(A)\right) . \\
& \text { 3. } \mathcal{Z} \text { AS A SET }
\end{aligned}
$$

This section is devoted to the study of the set

$$
\mathcal{Z}=\left\{(A, E): A \in L(\mathcal{H})^{+}, E \in \mathcal{Q}, A E=E^{*} A\right\},
$$

with the natural projections

$$
\begin{gathered}
p_{1}: \mathcal{Z} \longrightarrow L(\mathcal{H})^{+}, \quad p_{1}(A, E)=A \\
p_{2}: \mathcal{Z} \longrightarrow \mathcal{Q}, \quad p_{2}(A, E)=E
\end{gathered}
$$

and the corresponding fibers

$$
p_{1}^{-1}(\{A\}), \text { for } A \in L(\mathcal{H})^{+} ; p_{2}^{-1}(\{E\}) \text {, for } E \in \mathcal{Q} \text {. }
$$

Proposition 3.1. For every $T \in L(\mathcal{H})$ and $E \in \mathcal{Q}$, the pair $\left(T^{*} T, E\right)$ belongs to $\mathcal{Z}$ if and only if $T \mathcal{R}(E)$ is orthogonal to $T \mathcal{N}(E)$.

To prove this proposition we will use the following lemma.

Lemma 3.2. The following conditions are equivalent.

(1) The pair $(A, E)$ belongs to $\mathcal{Z}$.

(2) $\mathcal{N}(E) \subseteq A \mathcal{R}(E)^{\perp}$.

(3) $A \mathcal{N}(E) \subseteq \mathcal{N}\left(E^{*}\right)$.

(4) $A \mathcal{R}(E) \subseteq \mathcal{R}\left(E^{*}\right)$.

Proof. $1 \leftrightarrow 2$ : Suppose that the pair $(A, E) \in \mathcal{Z}$ then $A E=E^{*} A$. If $x \in \mathcal{N}(E)$ then $0=A E x=E^{*} A x$. Hence $A x \in \mathcal{N}\left(E^{*}\right)=\mathcal{R}(E)^{\perp}$, or equivalently, $x \in$ $A^{-1}\left(\mathcal{R}(E)^{\perp}\right)=A(\mathcal{R}(E))^{\perp}$. Then $\mathcal{N}(E) \subseteq A(\mathcal{R}(E))^{\perp}$.

Conversely, if $\mathcal{N}(E) \subseteq A \mathcal{R}(E)^{\perp}$, then by taking orthogonal complement we get $\overline{A \mathcal{R}(E)} \subseteq \mathcal{R}\left(E^{*}\right)$. Therefore $\mathcal{R}(A E) \subseteq \mathcal{R}\left(E^{*}\right)$, which implies $A E=E^{*} A E$, and this shows that $A E$ is Hermitian, i.e., $A E=E^{*} A$.

From this identity $A \mathcal{R}(E)^{\perp}=A^{-1}\left(\mathcal{R}(E)^{\perp}\right)$ the equivalences $2 \leftrightarrow 3$ and $3 \leftrightarrow 4$ are straightforward.

Proof. (of the proposition) Let $T=U A^{1 / 2}$ be the polar decomposition of $T$ with $A=T^{*} T$ and $U$ a partial isometry from $\overline{\mathcal{R}\left(A^{1 / 2}\right)}$ onto $\overline{\mathcal{R}(T)}$. If $(A, E) \in$ $\mathcal{Z}$ then, by the previous lemma, $\mathcal{H}=\mathcal{R}(E) \dot{+} \mathcal{N}(E) \subseteq \mathcal{R}(E)+A^{-1}\left(\mathcal{R}(E)^{\perp}\right)$. Applying $A^{1 / 2}$ to both sides of the equality, $\mathcal{R}\left(A^{1 / 2}\right)=A^{1 / 2} \mathcal{R}(E)+A^{1 / 2} \mathcal{N}(E)$ and $A^{1 / 2} \mathcal{N}(E) \subseteq A^{1 / 2} A^{-1}\left(\mathcal{R}(E)^{\perp}\right) \subseteq A^{-1 / 2}\left(\mathcal{R}(E)^{\perp}\right)=A^{1 / 2} \mathcal{R}(E)^{\perp}$. Therefore the sum is orthogonal, i.e., $\mathcal{R}\left(A^{1 / 2}\right)=A^{1 / 2} \mathcal{R}(E) \oplus A^{1 / 2} \mathcal{N}(E)$. Finally, applying $U$ to this equality we get that $\mathcal{R}(T)=T \mathcal{R}(E) \oplus T \mathcal{N}(E)$ because $U$ preserves orthogonality when applied to subsets of $\mathcal{R}\left(A^{1 / 2}\right)$.

Conversely, if $T \mathcal{R}(E)$ is orthogonal to $T \mathcal{N}(E)$ then $\mathcal{R}(T)=T \mathcal{R}(E) \oplus T \mathcal{N}(E)$. Therefore, $T \mathcal{N}(E)=T \mathcal{R}(E)^{\perp} \cap \mathcal{R}(T)$ and then $\mathcal{N}(E)=T^{-1}\left(T \mathcal{R}(E)^{\perp} \cap \mathcal{R}(T)\right)=$ $T^{-1}\left(T \mathcal{R}(E)^{\perp}\right)=T^{-1}\left(T^{*-1}\left(\mathcal{R}(E)^{\perp}\right)\right) \subseteq\left(T^{*} T\right)^{-1}\left(\mathcal{R}(E)^{\perp}\right)$. 
Remark 3.3. As we have shown in the proof of the proposition above, $\left(T^{*} T, E\right) \in$ $\mathcal{Z}$ if and only if $T \mathcal{R}(E) \oplus T \mathcal{N}(E)=\mathcal{R}(T)$; in particular, $(A, E) \in \mathcal{Z}$ if and only if $A^{1 / 2} \mathcal{R}(E) \oplus A^{1 / 2} \mathcal{N}(E)=\mathcal{R}\left(A^{1 / 2}\right)$. Observe that the subspaces $T \mathcal{R}(E)$ and $T \mathcal{N}(E)$ (resp. $A^{1 / 2} \mathcal{R}(E), A^{1 / 2} \mathcal{N}(E)$ ) need not to be closed. However, if $T$ is invertible, then, obviously, $T \mathcal{R}(E)$ and $T \mathcal{N}(E)$ are closed and it is easy to see that $\left(T^{*} T, E\right) \in \mathcal{Z}$ if and only if $T \mathcal{R}(E) \oplus T \mathcal{N}(E)=\mathcal{H}$.

We collect some facts about the projections $p_{1}$ and $p_{2}$.

Proposition 3.4. The following assertions hold:

(1) For every $E \in \mathcal{Q}, \theta(E)=E^{*} E+\left(I-E^{*}\right)(I-E) \in G L(\mathcal{H})^{+}$and the mapping $s(E)=(\theta(E), E)$ verifies $s(E) \in \mathcal{Z}$ for all $E \in \mathcal{Q}$ and $p_{2} \circ s=I_{\mathcal{Q}}$ (i.e. $s$ is a global section of $\left.p_{2}\right)$.

(2) $p_{1}: \mathcal{Z} \longrightarrow L(\mathcal{H})^{+}$is surjective.

(3) $p_{2}: \mathcal{Z} \longrightarrow \mathcal{Q}$ is surjective.

(4) For every $E \in \mathcal{Q}, p_{2}^{-1}(\{E\})$ is convex.

Proof. 1. It is easy to see that $\mathcal{N}(\theta(E))=\mathcal{N}(E) \cap \mathcal{R}(E)=\{0\}$ so that $\theta(E)$ is injective. To see that $\theta(E)$ is surjective observe that $\mathcal{R}\left(E^{*} E\right) \subseteq \mathcal{R}(\theta(E))$. Since $\mathcal{R}(E)$ is closed, $\mathcal{R}\left(E^{*} E\right)=E^{*}(\mathcal{R}(E))=E^{*}\left(\mathcal{N}\left(E^{*}\right)^{\perp}\right)=\mathcal{R}\left(E^{*}\right)$. Then $\mathcal{R}\left(E^{*}\right) \subseteq \mathcal{R}(\theta(E))$. In a similar way, $\mathcal{N}\left(E^{*}\right)=\mathcal{R}\left(I-E^{*}\right) \subseteq \mathcal{R}(\theta(E))$. Then $\mathcal{R}(\theta(E))=\mathcal{H}$.

The rest of the assertion follows easily.

2. Given $A \in L(\mathcal{H})^{+}$, the pair $(A, I) \in \mathcal{Z}$ and $p_{1}(A, I)=A$.

3. Given $E \in \mathcal{Q}$, by 1 , the pair $(\theta(E), E) \in \mathcal{Z}$ and $p_{2}(\theta(E), E)=E$.

From now on, we identify the fiber $p^{-1}(\{A\})=\{(A, E):(A, E) \in \mathcal{Z}\}$ with

$$
\mathcal{Z}_{A}:=\left\{E \in \mathcal{Q}: A E=E^{*} A\right\}
$$

and $p_{2}^{-1}(\{E\})=\{(A, E):(A, E) \in \mathcal{Z}\}$ with

$$
\mathcal{Z}^{E}:=\left\{A \in L(\mathcal{H})^{+}: A E=E^{*} A\right\}
$$

The next two sections are devoted to the characterization of fibers $\mathcal{Z}_{A}$ and $\mathcal{Z}^{E}$ for any $A \in L(\mathcal{H})^{+}, E \in \mathcal{Q}$. Thus, for $A \in L(\mathcal{H})^{+}$the set $\mathcal{Z}_{A}$ contains all oblique projections which are "orthogonalized" by $A$, and, for $E \in \mathcal{Q}$ the set $\mathcal{Z}^{E}$ contains all positive operators which "orthogonalize" $E$.

\section{THE $\operatorname{SeT} \mathcal{Z}_{A}$}

Observe that

$$
\mathcal{Z}_{A}=\bigcup_{\mathcal{S}} \mathcal{P}(A, \mathcal{S})
$$

where $\mathcal{S}$ runs over $G r$. Of course, a given $\mathcal{S}$ adds some projections only if $A$ and $\mathcal{S}$ are compatible. Notice also that it is a disjoint union.

The following subset of $\mathcal{P}$ is helpful to characterize the set $\mathcal{Z}_{A}$. Define

$$
\mathcal{P}_{A}=\left\{P \in \mathcal{P}: \mathcal{R}\left(P A^{1 / 2}\right) \subseteq \mathcal{R}\left(A^{1 / 2}\right)\right\}
$$


Observe that $P \in \mathcal{P}_{A}$ if and only if the equation $P A^{1 / 2}=A^{1 / 2} X$ admits a solution.

For $A \in L(\mathcal{H})^{+}$denote by $P_{A}$ the orthogonal projection onto $\overline{\mathcal{R}(A)}$.

If $P \in \mathcal{P}_{A}$ then $P\left(\mathcal{R}\left(A^{1 / 2}\right)\right) \subseteq \overline{\mathcal{R}(A)}$ so that $P(\overline{\mathcal{R}(A)}) \subseteq \overline{\mathcal{R}(A)}$ and then $P P_{A}=P_{A} P P_{A}$. Therefore, $P P_{A}$ is positive; in particular $P P_{A}$ and $P\left(I-P_{A}\right)$ are both (orthogonal) projections and $P=P P_{A}+P\left(I-P_{A}\right)$. Hence, the projections of $\mathcal{P}_{A}$ can be written as the sum of two orthogonal projections, one with range included in $\overline{\mathcal{R}(A)}$ and the other with range included in $\mathcal{N}(A)$.

Theorem 4.1. Given $A \in L(\mathcal{H})^{+}$,

$\mathcal{Z}_{A}=\left\{\left[\begin{array}{cc}E_{1} & 0 \\ W & E_{2}\end{array}\right]: E_{1}=\left.A^{1 / 2^{\dagger}} P A^{1 / 2}\right|_{\overline{\mathcal{R}(A)}}, P \in \mathcal{P}_{A}, E_{2}^{2}=E_{2}, E_{2} W=W\left(I-E_{1}\right)\right\}$,

where the matrix representation is that induced by the decomposition $\mathcal{H}=\overline{\mathcal{R}(A)} \oplus$ $\mathcal{N}(A)$.

The proof of this theorem relies on the following lemmas.

Lemma 4.2. Let $A \in L(\mathcal{H})^{+}$and $E$ an $A$-selfadjoint projection. Then $P_{A} E$ and $E\left(I-P_{A}\right)$ are A-selfadjoint projections.

Proof. If $A E=E^{*} A$ then, $A P_{A} E=E^{*} P_{A} A=\left(P_{A} E\right)^{*} A$ and $P_{A} E$ is $A$-selfadjoint. Since $P_{A} E=A^{\dagger} E^{*} A$, it follows that $P_{A} E=P_{A} E P_{A}$. Then $\left(P_{A} E\right)^{2}=P_{A} E P_{A} E=$ $P_{A} E^{2}=P_{A} E$.

Also $\left(I-P_{A}\right) E\left(I-P_{A}\right)=\left(E-P_{A} E\right)\left(I-P_{A}\right)=\left(E-P_{A} E P_{A}\right)\left(I-P_{A}\right)=E(I-$ $\left.P_{A}\right)$. Then, $E\left(I-P_{A}\right)$ is a projection and $A E\left(I-P_{A}\right)=A\left(I-P_{A}\right) E\left(I-P_{A}\right)=0$, so that it is $A$-selfadjoint.

Lemma 4.3. Let $A \in L(\mathcal{H})^{+}$. Then, $E$ is an $A$-selfadjoint projection if and only if

$$
E=\left[\begin{array}{cc}
E_{1} & 0 \\
W & E_{2}
\end{array}\right]
$$

where $E_{1} \in L(\overline{\mathcal{R}(A)})$ is an $A$-selfadjoint projection, $E_{2} \in L(\mathcal{N}(A))$ is an oblique projection and $W \in L(\mathcal{R}(A), \mathcal{N}(A))$ satisfies that $E_{2} W=W\left(I-E_{1}\right)$.

Proof. Suppose that $E$ is a projection such that $A E=E^{*} A$, then, by Lemma $4.2 P_{A} E=P_{A} E P_{A}, E\left(I-P_{A}\right)=\left(I-P_{A}\right) E\left(I-P_{A}\right)$ so that $E=P_{A} E+E(I-$ $\left.P_{A}\right)+\left(I-P_{A}\right) E P_{A}=\left[\begin{array}{cc}E_{1} & 0 \\ W & E_{2}\end{array}\right]$, where $E_{1}=\left.P_{A} E\right|_{\overline{\mathcal{R}(A)}}, E_{2}=\left.E\left(I-P_{A}\right)\right|_{\mathcal{N}(A)}$ and $W=\left.\left(I-P_{A}\right) E P_{A}\right|_{\overline{\mathcal{R}(A)}}$. By Lemma 4.2, $E_{1}$ and $E_{2}$ are $A$-selfadjoint projections. It is easy to see that $E_{2} W=W\left(I-E_{1}\right)=\left.\left(E P_{A}-E P_{A} E\right)\right|_{\overline{\mathcal{R}(A)}}$.

Conversely, if $E=\left[\begin{array}{cc}E_{1} & 0 \\ W & E_{2}\end{array}\right]$, with $E_{1}$ and $E_{2}$ projections and $E_{2} W=W(I-$ $E_{1}$ ), then $E^{2}=\left[\begin{array}{cc}E_{1} & 0 \\ W P+E_{2} W & E_{2}\end{array}\right]=E$ and $E$ is a projection. Also, $A E=$ $\left[\begin{array}{cc}A E_{1} & 0 \\ 0 & 0\end{array}\right]=\left[\begin{array}{cc}E_{1}^{*} A & 0 \\ 0 & 0\end{array}\right]=E^{*} A$, because $E_{1}$ is $A$-selfadjoint. 
Lemma 4.4. Let $A \in L(\mathcal{H})^{+}$and $E \in \mathcal{Q}$ such that $R(E) \subseteq \overline{\mathcal{R}(A)}$. Then, $E$ is $A$-selfadjoint if and only if $E=A^{1 / 2^{\dagger}} P A^{1 / 2}$, where $P \in \mathcal{P}_{A}$.

Proof. Using Douglas' theorem, it is easy to verify that if $P \in \mathcal{P}_{A}$ then $E=$ $A^{1 / 2^{\dagger}} P A^{1 / 2}$ is well defined and is a bounded $A$-selfadjoint projection. Conversely, applying Proposition 3.4 of [7] the converse follows immediately.

In particular, if $A$ has closed range, the $A$-selfadjoint projections such $R(E) \subseteq$ $\mathcal{R}(A)$, are given by $E=A^{1 / 2^{\dagger}} P A^{1 / 2}$, where $P \in \mathcal{P}$ verifies that $R(P) \subseteq \mathcal{R}(A)$.

Lemma 4.5. Let $A \in L(\mathcal{H})^{+}$. Then $E \in \mathcal{Z}_{A} \cap \mathcal{P}$ if and only if $E=E_{1}+E_{2}$, where $E_{1}, E_{2} \in \mathcal{P}, \mathcal{R}\left(E_{1}\right) \subseteq \overline{\mathcal{R}(A)}, \mathcal{R}\left(E_{2}\right) \subseteq \mathcal{N}(A)$ and $\mathcal{R}\left(A E_{1}\right) \subseteq \mathcal{R}\left(E_{1}\right)$.

Proof. Suppose that $E=E_{1}+E_{2}$, with $E_{1}, E_{2} \in \mathcal{P}, \mathcal{R}\left(E_{1}\right) \subseteq \overline{\mathcal{R}(A)}, \mathcal{R}\left(E_{2}\right) \subseteq$ $\mathcal{N}(A)$ and $\mathcal{R}\left(A E_{1}\right) \subseteq \mathcal{R}\left(E_{1}\right)$. Then $E_{1} E_{2}=0=E_{2} E_{1}$ so that $E^{2}=E=E^{*}$. To see that $E \in \mathcal{Z}_{A}$, observe that $A E=A E_{1}=E_{1} A E_{1}$, because $\mathcal{R}\left(E_{2}\right) \subseteq \mathcal{N}(A)$ and $\mathcal{R}\left(A E_{1}\right) \subseteq \mathcal{R}\left(E_{1}\right)$. Then $A E$ is positive so that $A E=E^{*} A$.

Conversely, if $E \in \mathcal{Z}_{A}$, by Lemma 4.3,

$$
E=\left[\begin{array}{cc}
E_{1} & 0 \\
W & E_{2}
\end{array}\right]
$$

where $E_{1} \in L(\overline{\mathcal{R}(A)})$ is an $A$-selfadjoint projection, $E_{2} \in L(\mathcal{N}(A))$ is an oblique projection and $W \in L(\mathcal{R}(A), \mathcal{N}(A))$ satisfies that $E_{2} W=W\left(I-E_{1}\right)$. If $E$ belongs also to $\mathcal{P}$ then $E_{1}^{*}=E_{1}, E_{2}^{*}=E_{2}$ and $W=0$. Then $E=E_{1}^{\prime}+E_{2}^{\prime}$, where $E_{1}^{\prime}$ and $E_{2}^{\prime}$ are the orthogonal projections acting on $\mathcal{H}$ defined by $E_{1}^{\prime}=E_{1} P_{A}$ and $E_{2}^{\prime}=E_{2}\left(I-P_{A}\right)$, with $P_{A}$ the orthogonal projection onto $\overline{\mathcal{R}(A)}$. Finally $E$ is $A$-selfadjoint if and only if $A E=E A$ or equivalently $A E_{1}^{\prime}=E_{1}^{\prime} A$ because $A E_{2}^{\prime}=0=E_{2}^{\prime} A$. Hence $\mathcal{R}\left(A E_{1}^{\prime}\right) \subseteq \mathcal{R}\left(E_{1}^{\prime}\right)$.

Proposition 4.6. Let $A \in L(\mathcal{H})^{+}$, and $B \in G L(H)^{+}$such that $\mathcal{Z}_{A} \subseteq \mathcal{Z}_{B}$, then there exists $\lambda>0$ such that $B=\lambda A$. In particular $A$ is invertible and $\mathcal{Z}_{A}=\mathcal{Z}_{B}$.

Proof. First consider $B=I$. In this case $\mathcal{Z}_{B}=\mathcal{P}$. Suppose that $\mathcal{Z}_{A} \subseteq \mathcal{P}$. If $A=0$ then $\mathcal{Z}_{A}=\mathcal{Q}$ which is not included in $\mathcal{P}$; therefore $A \neq 0$. Moreover $A$ must be injective. Suppose on the contrary that $\mathcal{N}(B) \neq\{0\}$ and consider the operator $E$ defined by its matrix decomposition in terms of $\overline{\mathcal{R}(A)}$ and $\mathcal{N}(A)$ as $E=\left[\begin{array}{cc}0 & 0 \\ W & I\end{array}\right]$, where $W \neq 0$ ( this is possible because $\mathcal{R}(A) \neq 0$ and $\mathcal{N}(A) \neq 0$ ). It is easy to see that $E$ is a projection and $A E=0=E^{*} A$ so that $E$ is $A$ selfadjoint, but $E \notin \mathcal{P}$. Let us see that $A$ si also surjective: if $x \in \mathcal{H}, x \neq 0$, then the pair $(A,[x])$ is compatible because $[x]$ is finite dimensional. Since $A$ is injective there exists only one projection onto $[x]$ which is $A$-selfadjoint and it is given by $P_{A,[x]}=P_{[x] / / A[x]}$. Using that $\mathcal{Z}_{A} \subseteq \mathcal{P}$, it must hold that $A[x]^{\perp}=[x]^{\perp}$, or equivalently, $A[x]=[x]$. Therefore, there exists $\lambda$ such that $A x=\lambda x$ so that $x \in \mathcal{R}(A)$. Then $\mathcal{R}(A)=\mathcal{H}$ and $A$ is invertible. In this case, by Theorem 4.1, the set $\mathcal{Z}_{A}=\left\{A^{-1 / 2} P A^{1 / 2}: P \in \mathcal{P}\right\}=A^{-1 / 2} \mathcal{P} A^{1 / 2}$. If $\mathcal{Z}_{A} \subseteq \mathcal{P}$, for every $P \in \mathcal{P}$, 
$A^{-1 / 2} P A^{1 / 2} \in \mathcal{P}$. Hence, $A^{-1 / 2} P A^{1 / 2}=A^{1 / 2} P A^{-1 / 2}$ so that $P A=A P$ for every $P \in \mathcal{P}$. By [11], there exists $\lambda>0$ such that $A=\lambda I$.

For any $B \in G L(\mathcal{H})^{+}$the condition $\mathcal{Z}_{A} \subseteq \mathcal{Z}_{B}=B^{-1 / 2} \mathcal{P} B^{1 / 2}$ is equivalent to $B^{1 / 2} \mathcal{Z}_{A} B^{-1 / 2} \subseteq \mathcal{P}$. It holds that $B^{1 / 2} \mathcal{Z}_{A} B^{-1 / 2}=\mathcal{Z}_{B^{-1 / 2} A B^{-1 / 2}}$; hence $B^{-1 / 2} A B^{-1 / 2}=\lambda I$ or, equivalently $A=\lambda B$.

Denote by $\mathcal{Q}_{\mathcal{S}}$ the subset of $\mathcal{Q}$ of oblique projections with range $\mathcal{S}$.

Proposition 4.7. Let $A \in L(\mathcal{H})^{+}$.

(1) $\mathcal{Z}_{A} \cap \mathcal{Q}_{\mathcal{S}}=\mathcal{P}(A, \mathcal{S})$

(2) $\mathcal{Z}_{A} \cap \mathcal{Q}_{\mathcal{S}}$ is not empty if and only if $(A, \mathcal{S})$ is compatible.

(3) If $A \in G L(\mathcal{H})^{+}$then $\mathcal{Z}_{A} \cap \mathcal{Q}_{\mathcal{S}}=\left\{P_{A, \mathcal{S}}\right\}$.

(4) $\mathcal{Z}_{A} \cap \mathcal{Q}_{\mathcal{S}}$ contains a single element if and only if $(A, \mathcal{S})$ is compatible and $\mathcal{N}(A) \cap \mathcal{S}=\{0\}$.

\section{THE $\operatorname{SET} \mathcal{Z}^{E}$}

Given a fixed projection $E$ we look for the positive operators $A$ such that $E$ is $A$-selfadjoint, i.e., the set $\mathcal{Z}^{E}=\left\{A \in L(\mathcal{H})^{+}: A E=E^{*} A\right\}$. Observe that. by Proposition $3.4, \mathcal{Z}^{E}$ is a convex set.

Theorem 5.1. Given $E \in \mathcal{Q}$,

$$
\mathcal{Z}^{E}=\left\{A=A_{1}+A_{2}: A_{1}, A_{2} \in L(\mathcal{H})^{+}, \mathcal{R}\left(A_{1}\right) \subseteq \mathcal{R}\left(E^{*}\right), \mathcal{R}\left(A_{2}\right) \subseteq \mathcal{N}\left(E^{*}\right)\right\}
$$

Proof. Suppose that $A=A_{1}+A_{2}$, with $A_{1}$ and $A_{2}$ positive and such that $\mathcal{R}\left(A_{1}\right) \subseteq$ $\mathcal{R}\left(E^{*}\right), \mathcal{R}\left(A_{2}\right) \subseteq \mathcal{N}\left(E^{*}\right)$. Then $A$ is positive. To see that $E$ is $A$-selfadjoint, observe that $A E=\left(A_{1}+A_{2}\right) E=A_{1} E=E^{*} A_{1} E$, because $\mathcal{R}(E) \subseteq \mathcal{N}\left(A_{2}\right)$ and $\mathcal{R}\left(A_{1}\right) \subseteq \mathcal{R}\left(E^{*}\right)$. Noticing that $E^{*} A E$ is positive, $A E=E^{*} A E=E^{*} A$.

Conversely, if $A \in \mathcal{Z}^{E}$ then $A$ is positive and $A E=E^{*} A=E^{*} A E$ so that $\left(I-E^{*}\right) A E=0=E^{*} A(I-E)$. Therefore, $A=E^{*} A E+\left(I-E^{*}\right) A(I-E)$. If $A_{1}=E^{*} A E$ and $A_{2}=\left(I-E^{*}\right) A(I-E)$, then $A=A_{1}+A_{2}, A_{1}$ and $A_{2}$ are positive, $\mathcal{R}\left(A_{1}\right) \subseteq \mathcal{R}\left(E^{*}\right), \mathcal{R}\left(A_{2}\right) \subseteq \mathcal{N}\left(E^{*}\right)$.

Remark 5.2. By the above proposition, it is easy to see that

$$
\mathcal{Z}^{E}=\left\{A=A_{1} P_{\mathcal{R}\left(E^{*}\right)}+A_{2} P_{\mathcal{N}\left(E^{*}\right)}: A_{1} \in L\left(\mathcal{R}\left(E^{*}\right)\right)^{+}, A_{2} \in L\left(\mathcal{N}\left(E^{*}\right)\right)^{+}\right\} .
$$

Then the set $\mathcal{Z}^{E}$ can be identified with the product

$$
L\left(\mathcal{R}\left(E^{*}\right)\right)^{+} \times L\left(\mathcal{N}\left(E^{*}\right)\right)^{+}
$$

through the map

$$
\begin{aligned}
& \mathcal{Z}^{E} \longrightarrow L\left(\mathcal{R}\left(E^{*}\right)\right)^{+} \times L\left(\mathcal{N}\left(E^{*}\right)\right)^{+}, \\
& A \longrightarrow\left(\left.A E\right|_{\mathcal{R}\left(E^{*}\right)},\left.A(I-E)\right|_{\mathcal{N}\left(E^{*}\right)}\right)
\end{aligned}
$$

For every $E \in \mathcal{Q}$ the set $\mathcal{Z}^{E} \cap \mathcal{P}$ is not empty. In fact, $P=P_{\mathcal{N}(E)^{\perp}}$ has the same nullspace as $E$ and then $P E=P=E^{*} P$, which proves that $P \in \mathcal{Z}^{E} \cap \mathcal{P}$. The next proposition characterizes the set $\mathcal{Z}^{E} \cap \mathcal{P}$.

Proposition 5.3. Let $E \in \mathcal{Q}$. Then

$$
\mathcal{Z}^{E} \cap \mathcal{P}=\left\{P=P_{1}+P_{2}: P_{1}, P_{2} \in \mathcal{P}, P_{1} P_{2}=0, \mathcal{R}\left(P_{1}\right) \subseteq \mathcal{R}\left(E^{*}\right), \mathcal{R}\left(P_{2}\right) \subseteq \mathcal{N}\left(E^{*}\right)\right\}
$$


Proof. If $P=P_{1}+P_{2}$ with $P_{i} \in \mathcal{P}, \mathrm{i}=1,2, P_{1} P_{2}=0, \mathcal{R}\left(P_{1}\right) \subseteq \mathcal{R}\left(E^{*}\right)$ and $\mathcal{R}\left(P_{2}\right) \subseteq \mathcal{N}\left(E^{*}\right)$ then $P$ is an orthogonal projection. To see that $P \in \mathcal{Z}^{E}$ observe that $E^{*} P=E^{*}\left(P_{1}+P_{2}\right)=P_{1}=P_{1}^{*}=P E$.

Conversely, if $P \in \mathcal{Z}^{E} \cap \mathcal{P}$ then, by Proposition 5.1 $P=A_{1}+A_{2}$, with $A_{1}$ and $A_{2}$ positive, $\mathcal{R}\left(A_{1}\right) \subseteq \mathcal{R}\left(E^{*}\right)$ and $\mathcal{R}\left(A_{2}\right) \subseteq \mathcal{N}\left(E^{*}\right)$. From $P^{2}=P$ it follows that

$$
A_{1}^{2}+A_{2}^{2}+A_{1} A_{2}+A_{2} A_{1}=A_{1}+A_{2} .
$$

Multiplying to the left by $E^{*}$ and noticing that $E^{*} A_{1}=A_{1}=A_{1} E$ and $E^{*} A_{2}=$ $0=A_{2} E$ it follows that $A_{1}^{2}+A_{1} A_{2}=A_{1}$; multiplying by $E$ to the right, $A_{1}^{2}=A_{1}$. In a similar way, $A_{2}^{2}=A_{2}$. Therefore $A_{1}$ and $A_{2}$ are orthogonal projections because they are positive. It follows from (5.1) that $A_{1} A_{2}+A_{2} A_{1}=0$, then $A_{1} A_{2}=0=A_{2} A_{1}$.

It is an old result by Penrose [20] and Greville [10] ( see also an infinite dimensional treatment in [4]) that every $Q \in \mathcal{Q}$ can be decomposed as the MoorePenrose inverse of a product of two orthogonal projections. More precisely, if $E$ is the oblique projection with $\mathcal{R}(E)=\mathcal{W}$ and $\mathcal{N}(E)=\mathcal{M}^{\perp}$ then $E^{\dagger}=P_{\mathcal{M}^{\perp}} P_{\mathcal{W}}$ and therefore, $E=\left(P_{\mathcal{M}^{\perp}} P_{\mathcal{W}}\right)^{\dagger}$. Thus

$\mathcal{Z}^{E} \cap \mathcal{P}=\left\{P \in \mathcal{P}: P E=E^{*} P\right\}=\left\{P_{\mathcal{N}} \in \mathcal{P}: P_{\mathcal{N}}\left(P_{\mathcal{M}^{\perp}} P_{\mathcal{W}}\right)^{\dagger}=\left(P_{\mathcal{W}} P_{\mathcal{M}^{\perp}}\right)^{\dagger} P_{\mathcal{N}}\right\}$

The next two propositions exhibit a sort of disjoint behaviour of the fibers $\mathcal{Z}^{E}$.

Proposition 5.4. Let $E, F \in \mathcal{Q}$. If $\mathcal{Z}^{E} \subseteq \mathcal{Z}^{F}$ then $E F=0$ or $E(I-F)=0$.

Proof. Suppose first that $E$ and $F$ are selfadjoint projections. If $\mathcal{Z}^{E} \subseteq \mathcal{Z}^{F}$ then $E \in \mathcal{Z}^{F}$ because $E \in \mathcal{Z}^{E}$. Therefore $E F=F E$. In this case, $E F=P_{\mathcal{R}(E) \cap \mathcal{R}(F)}$, $E(I-F)=P_{\mathcal{R}(E) \cap \mathcal{N}(F)}$ and $E=E F+E(I-F)$.

Suppose that $E F$ and $E(I-F)$ are both different from zero; let $x \in \mathcal{R}(E) \cap$ $\mathcal{N}(F),\|x\|=1$, and $y \in \mathcal{R}(E) \cap \mathcal{R}(F),\|y\|=1 / 2$ and define $B x=y$ and $B=0$ in the orthogonal complement of $x,[y]^{\perp}$. The operator $B$ is bounded and $\|B\|=1 / 2$; also $\mathcal{R}(B)=[y] \subseteq \mathcal{R}(E)$; then $\mathcal{R}(E)^{\perp} \subseteq[y]^{\perp}=\mathcal{N}(B)$. Therefore, $\mathcal{R}\left(B^{*}\right)=\overline{\mathcal{R}\left(B^{*}\right)}=\mathcal{N}(B)^{\perp} \subseteq \mathcal{R}(E)$. Define $A=E+B+B^{*}$; then $A$ is selfadjoint, $\mathcal{R}(A) \subseteq \mathcal{R}(E)$. In fact $A$ is positive: first observe that $B+B^{*}=$ $E\left(B+B^{*}\right) E$ because $\mathcal{R}\left(B+B^{*}\right) \subseteq R(E)$; then for $x \in \mathcal{H},\left|\left\langle\left(B+B^{*}\right) x, x\right\rangle\right|=$ $\left|\left\langle\left(B+B^{*}\right) E x, E x\right\rangle\right| \leq 2\|B\|\|E x\|^{2}=\|E x\|^{2}$. Therefore $|\langle A x, x\rangle|=\mid(E+B+$ $\left.\left.B^{*}\right) x, x\right\rangle \mid \geq\|x\|^{2}-\|E x\|^{2}=0$.

Observe that $L(\mathcal{R}(E))^{+} \subseteq \mathcal{Z}^{E}$; in particular $A \in \mathcal{Z}^{E}$. But $A \notin \mathcal{Z}^{F}$ : since $F=F^{*}$, a positive operator $A \in \mathcal{Z}^{F}$ if and only if $F A(I-F)=0$ but, in this case, $F A(I-F)=F\left(E+B+B^{*}\right)(I-F)=F B(I-F)=B \neq 0$.

Therefore, $E F=0$ or $E(I-F)=0$, which ends the proof in the special case where $E$ and $F$ are orthogonal projections.

In the general case, observe that if $F$ is an oblique projection and we consider an invertible $A \in \mathcal{Z}^{F}$, then, by the comment above, $B \in \mathcal{Z}^{F}$ if and only if $A^{-1 / 2} B A^{-1 / 2} \in \mathcal{Z}^{A^{1 / 2} F A^{-1 / 2}}$, where $A^{1 / 2} F A^{-1 / 2} \in \mathcal{P}$, i. e., $A^{-1 / 2} \mathcal{Z}^{F} A^{-1 / 2}=$ $\mathcal{Z}^{A^{1 / 2} Q A^{-1 / 2}}$. Let $E$ and $F$ be oblique projections such that $\mathcal{Z}^{E} \subseteq \mathcal{Z}^{F}$ and consider an invertible $A \in \mathcal{Z}^{E}$. Then $A \in Z^{F}$ so that $\mathcal{Z}^{E} \subseteq \mathcal{Z}^{F}$, i.e., $\mathcal{Z}^{A^{1 / 2} E A^{-1 / 2}} \subseteq$ $\mathcal{Z}^{A^{1 / 2} F A^{-1 / 2}}$. But this last inclusion implies that $A^{1 / 2} E A^{-1 / 2}=A^{1 / 2} E F A^{-1 / 2}$ 
or $A^{1 / 2} E A^{-1 / 2}=A^{1 / 2} E(I-F) A^{-1 / 2}$, by the first part, and cancelling the $A^{\prime} \mathrm{s}$, $E=E F$ or $E=E(I-F)$.

Corollary 5.5. Let $E, F \in \mathcal{Q}$ such that $E \neq 0$ and $F \neq I$. If $\mathcal{Z}^{E} \subseteq \mathcal{Z}^{F}$ then $F=E$ or $F=I-E$, so that $\mathcal{Z}^{E}=\mathcal{Z}^{F}$.

Proof. As in the proof of the above proposition, we can suppose that $E, F \in \mathcal{P}$. If $\mathcal{Z}^{E} \subseteq \mathcal{Z}^{F}$ then by Proposition $5.4, E=E F$ or $E F=0$. Since $\mathcal{Z}^{E}=\mathcal{Z}^{(I-E)}$, we deduce that $I-E=(I-E) F$ or $(I-E) F=0$. If $E=E F$ and $I-E=(I-E) F$ then $F=I$. If $E=E F$ and $(I-E) F=0$ then $E=F$. If $E F=0$ and $I-E=(I-E) F$ then $F=I-E$. Finally, if $E F=0$ and $(I-E) F=0$ then $F=0$.

Proposition 5.6. Consider $A \in L(\mathcal{H})^{+}$and $E \in \mathcal{Q}$, such that the representations of $A$ and $E$ induced by the decomposition $\mathcal{H}=\overline{\mathcal{R}(E)} \oplus \mathcal{R}(E)^{\perp}$ are $A=\left[\begin{array}{cc}a & b \\ b^{*} & c\end{array}\right]$ and $E=\left[\begin{array}{ll}1 & r \\ 0 & 0\end{array}\right]$ respectively. Then the following conditions are equivalent.

(1) $A \in \mathcal{Z}^{E} \cap \mathcal{Z}^{E^{*}}$.

(2) $A E=E A=A E^{*}$.

(3) $A=A_{1}+A_{2}$ with $A_{1}, A_{2} \in L(\mathcal{H})^{+}$such that

$$
\mathcal{R}\left(A_{1}\right) \subseteq \mathcal{R}(E) \cap \mathcal{N}(E)^{\perp}, \quad \mathcal{R}\left(A_{2}\right) \subseteq \mathcal{R}(E)^{\perp} \cap \mathcal{N}(E)
$$

(4) $A=\left[\begin{array}{ll}a & 0 \\ 0 & c\end{array}\right]$ with ar $=r c=0$.

Proof. $1 \rightarrow 2: A \in \mathcal{Z}^{E} \cap \mathcal{Z}^{E^{*}}$ if and only if $A E=E^{*} A$ and $A E^{*}=E A$. Hence $A^{2} E=A E^{*} A=E A^{2}$. Since $A$ is positive it follows that $A E=E A$ and the first equality holds. Taking adjoint to the last equality $A$ and $E^{*}$ also commute. Therefore $A E=E^{*} A=A E^{*}$ and the second equality follows.

$2 \rightarrow 3$ : If $A E=E A=A E^{*}$ then $A E^{*}=E^{*} A$ and $A \in \mathcal{Z}^{E^{*}}$. By Proposition 5.1, $A \in \mathcal{Z}^{E^{*}}$ if and only if $A=A_{1}+A_{2}$, with $A_{1}, A_{2} \in L(\mathcal{H})^{+}, \mathcal{R}\left(A_{1}\right) \subseteq \mathcal{R}(E)$ and $\mathcal{R}\left(A_{2}\right) \subseteq \mathcal{N}(E)$. Also $A E=E A$ if and only if $A_{1} E+A_{2} E=E A_{1}$ because $E A_{1}=A_{1}$ and $E A_{2}=0$, or equivalently $A_{2} E=A_{1}(I-E)$. Therefore $A_{2} E=0$ and $A_{1}(I-E)=0$ because $\mathcal{R}\left(A_{1}\right) \cap \mathcal{R}\left(A_{2}\right)=\{0\}$. Hence $\mathcal{R}(E) \subseteq \mathcal{N}\left(A_{2}\right)$ and $\mathcal{N}(E) \subseteq \mathcal{N}\left(A_{1}\right)$. Then $\mathcal{R}\left(A_{2}\right) \subseteq \mathcal{N}(E) \cap \mathcal{R}(E)^{\perp}$ and $\mathcal{R}\left(A_{1}\right) \subseteq \mathcal{R}(E) \cap \mathcal{N}(E)^{\perp}$

$3 \rightarrow 4$ : if $A=A_{1}+A_{2}$ as in item 3 then $P A=P A P=A_{1}, P A(I-P)=0$ and $(I-P) A(I-P)=A_{2}$, where $P$ is the orthogonal projection onto $\mathcal{R}(E)$.

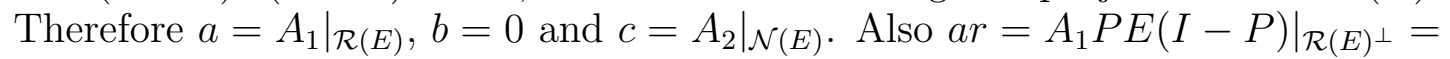
$\left.A_{1}(E-P)\right|_{\mathcal{R}(E)^{\perp}}=0$ because $A_{1}(E-P)=A_{1} E-A_{1} P=A_{1}-A_{1}=0$; the last equality follows from the fact that $\mathcal{R}(E)^{\perp}$ and $\mathcal{N}(E)$ are subsets of $\mathcal{N}\left(A_{1}\right)$. The equality $r c=0$ follows in a similar way.

$4 \rightarrow 1$ : It is straightforward.

Corollary 5.7. If $E \neq E^{*}$ then $\mathcal{Z}^{E} \cap \mathcal{Z}^{E^{*}}$ contains no (positive) invertible operator. More generally, $\mathcal{Z}^{E} \cap \mathcal{Z}^{E^{*}}$ contains no injective $A \in L(\mathcal{H})^{+}$. 
Remark 5.8. If $P \in \mathcal{P}$ then $\mathcal{Z}^{P}=\left\{A \in L(\mathcal{H})^{+}: A P=P A\right\}=\left(P^{\prime}\right)^{+}$, i.e., the cone of positive elements of the commutant algebra of $P$ in $L(\mathcal{H})$. Consequently,

$$
\bigcap_{P \in \mathcal{P}} \mathcal{Z}^{P}=\left\{\lambda I: \lambda \in \Re^{+}\right\}
$$

\section{ON THE TOPOLOGY OF $\mathcal{Z}$}

In order to study the topology of $\mathcal{Z}$, it is useful to consider the action of $G L(\mathcal{H})$ over $\mathcal{Z}$ given by

$$
\begin{gathered}
L: G L(\mathcal{H}) \times \mathcal{Z} \longrightarrow \mathcal{Z} \\
L(G,(A, E))=\left(G A G^{*}, G^{*-1} E G^{*}\right) .
\end{gathered}
$$

By restriction, $L$ defines actions over $\mathcal{Z}_{A}$ and $\mathcal{Z}^{E}$.

Lemma 6.1. For every $A \in L(\mathcal{H})^{+}, E \in \mathcal{Q}, G \in G L(\mathcal{H})$ it holds

$$
\begin{aligned}
& \mathcal{Z}_{G^{*-1} A G^{-1}}=G \mathcal{Z}_{A} G^{-1}, \\
& \mathcal{Z}^{G E G^{-1}}=G^{*-1} \mathcal{Z}^{E} G^{-1} .
\end{aligned}
$$

This shows that, by choosing convenient $A \in L(\mathcal{H})^{+}, E \in \mathcal{Q}$, one can get information on every $\mathcal{Z}_{A^{\prime}}, \mathcal{Z}^{E^{\prime}}$ for $E^{\prime}$ similar to $E$ and $A^{\prime}$ congruent to $A$, provided that we have that type of information on $\mathcal{Z}_{A}$ or $\mathcal{Z}^{E}$.

Observe that $\mathcal{Z}$ is a closed subset of $L(\mathcal{H})^{+} \times \mathcal{Q}$, if we provide $L(\mathcal{H})^{+} \times \mathcal{Q}$ with the induced topology of $L(\mathcal{H}) \times L(\mathcal{H})$.

Our main concerns deal with

$$
\mathcal{Z}^{\circ}=\left\{(A, E) \in \mathcal{Z}: A \in G L(\mathcal{H})^{+}\right\}
$$

with the fibers

$$
\mathcal{Z}_{A}^{\circ}=\left\{E \in \mathcal{Q}:(A, E) \in \mathcal{Z}^{\circ}\right\}=\mathcal{Z}_{A}
$$

and

$$
\left(\mathcal{Z}^{\circ}\right)^{E}=\left\{A \in G L(\mathcal{H})^{+}:(A, E) \in \mathcal{Z}^{\circ}\right\},
$$

for $A \in G L(\mathcal{H})^{+}$and $E \in \mathcal{Q}$.

Proposition 6.2. The mapping

$$
\begin{gathered}
\phi: \mathcal{Z}^{\circ} \longrightarrow G L(\mathcal{H})^{+} \times \mathcal{P} \\
(A, E) \longrightarrow\left(A, A^{1 / 2} E A^{-1 / 2}\right)
\end{gathered}
$$

is a homeomorphism with inverse

$$
\begin{gathered}
\psi: G L(\mathcal{H})^{+} \times \mathcal{P} \longrightarrow \mathcal{Z}^{\circ} \\
(A, P) \longrightarrow\left(A, A^{-1 / 2} E A^{1 / 2}\right) .
\end{gathered}
$$

Proof. It suffices to compute $\phi \circ \psi$ and $\psi \circ \phi$.

Consider the map

$$
\begin{gathered}
\alpha: G L(\mathcal{H})^{+} \times \mathcal{Q} \longrightarrow \mathcal{Q} \\
\alpha(A, E)=A^{1 / 2} E A^{-1 / 2} .
\end{gathered}
$$




\section{Proposition 6.3.}

$$
\alpha^{-1}(\mathcal{P})=\mathcal{Z}^{\circ}
$$

Proof. Suppose that $(A, E) \in \alpha^{-1}(\mathcal{P})$. Then $\alpha(A, E)=P \in \mathcal{P}$; this is equivalent to $A^{1 / 2} E A^{-1 / 2}=A^{-1 / 2} E^{*} A^{1 / 2}$. Therefore $A E=E^{*} A$ so that $(A, E) \in \mathcal{Z}^{\circ}$. This shows that $\alpha^{-1}(\mathcal{P}) \subseteq \mathcal{Z}^{\circ}$. Conversely, if $(A, E) \in \mathcal{Z}^{\circ}$ it has been shown that the projection $A^{1 / 2} E A^{-1 / 2}$ is selfadjoint, or equivalently, $\alpha(A, E) \in \mathcal{Z}^{\circ}$. This proves the other inclusion.

Denote again $\alpha=\left.\alpha\right|_{\mathcal{Z}^{\circ}}$.

Corollary 6.4. The map $\alpha: \mathcal{Z}^{\circ} \longrightarrow \mathcal{P}, \alpha(A, E)=A^{1 / 2} E A^{-1 / 2}=P_{\mathcal{R}\left(A^{1 / 2} E\right)}$ is continuous.

Proposition 6.5. For $A \in G L(\mathcal{H})^{+}$, consider the map $\alpha_{A}=\left.\alpha\right|_{\mathcal{Z}_{A}}$

$$
\begin{gathered}
\alpha_{A}: \mathcal{Z}_{A} \longrightarrow \mathcal{P} \\
\alpha_{A}(E)=A^{1 / 2} E A^{-1 / 2}
\end{gathered}
$$

is a homeomorphism, with inverse $\alpha_{A}^{-1}(P)=A^{-1 / 2} P A^{1 / 2}$.

Corollary 6.6. There is a natural bijection between the sets of connected components of $\mathcal{Z}^{\circ}$ and $\mathcal{P}$.

Proof. In fact, $\mathcal{Z}^{\circ}$ is homeomorphic to $G L(\mathcal{H})^{+} \times \mathcal{P}$ and $G L(\mathcal{H})^{+}$is contractible, by Kuiper's theorem [15]. Thus, $\mathcal{Z}^{\circ}$ has the homotopy type of $\mathcal{P}$. In particular, $\phi$ induces a bijection between the set of connected components of $\mathcal{Z}^{\circ}$ and that of $\mathcal{P}$.

We extend the map $\alpha: \mathcal{Z}^{\circ} \longrightarrow \mathcal{P}$ to $\mathcal{Z}$, but we are obliged to loose the continuity. The reason behind this lost is the fact that inversion can not be extended with continuity from $G L(\mathcal{H})$.

Proposition 6.7. Let $(A, E) \in \mathcal{Z}$. Then $P=A^{1 / 2^{\dagger}} E^{*} A^{1 / 2}$ is well defined and $P \in \mathcal{P}$. Moreover, $P=P_{\mathcal{M}}$, where $\mathcal{M}=\overline{\mathcal{R}\left(A^{1 / 2} E\right)}$.

Proof. If $(A, E) \in \mathcal{Z}$ then, by Lemma $4.2, P_{A} E \in \mathcal{Z}_{A}$ and $\mathcal{R}\left(P_{A} E\right) \subseteq \overline{\mathcal{R}(A)}$. In this case, applying Proposition 3.4 of [7], $P_{\mathcal{M}}\left(\mathcal{R}\left(A^{1 / 2}\right)\right) \subseteq \mathcal{R}\left(A^{1 / 2}\right)$ and $P_{A} E=$ $A^{1 / 2^{\dagger}} P_{\mathcal{M}} A^{1 / 2}$, where $\mathcal{M}=\overline{\mathcal{R}\left(A^{1 / 2} E\right)}$. Notice that the Moore-Penrose inverse of $A$ is not necessarily bounded and its domain is the (dense) set $\mathcal{R}\left(A^{1 / 2}\right) \oplus$ $\mathcal{N}(A)$. Therefore, $A^{1 / 2} E=A^{1 / 2} P_{A} E=P_{\mathcal{M}} A^{1 / 2}$, or $E^{*} A^{1 / 2}=A^{1 / 2} P_{\mathcal{M}}$. Hence, $A^{1 / 2}{ }^{\dagger} E^{*} A^{1 / 2}=P_{A} P_{\mathcal{M}}=P_{\mathcal{M}}$

In view of Proposition 6.7, we can extend $\alpha$ to $\mathcal{Z}$, in the following way:

$$
\tilde{\alpha}: \mathcal{Z} \longrightarrow \mathcal{P}, \tilde{\alpha}(A, E)=A^{1 / 2^{\dagger}} E^{*} A^{1 / 2}=P_{\overline{\mathcal{R}\left(A^{1 / 2} E\right)}},
$$

where $(A, E) \in \mathcal{Z}$. However, $\tilde{\alpha}$ is no longer continuous. 
Proposition 6.8. For $A \in L(\mathcal{H})^{+}$consider $\tilde{\alpha}_{A}: \mathcal{Z}_{A} \longrightarrow \mathcal{P}, \tilde{\alpha}_{A}(E)=\tilde{\alpha}(A, E)=$ $P_{\overline{\mathcal{R}\left(A^{1 / 2} E\right)}}$. Then $\tilde{\alpha}_{A}\left(\mathcal{Z}_{A}\right)=\left\{P \in \mathcal{P}_{A}: \mathcal{R}(P) \subseteq \overline{\mathcal{R}(A)}\right\}$. In particular, if $A$ has closed range then $\tilde{\alpha}_{A}\left(\mathcal{Z}_{A}\right)=\{P \in \mathcal{P}: \mathcal{R}(P) \subseteq \mathcal{R}(A)\}$.

For a fixed $(I, P) \in \mathcal{Z}^{\circ}$ define the map

$$
\pi: G L(\mathcal{H}) \longrightarrow \mathcal{Z}^{\circ}, \pi(G)=L(G,(I, P))=\left(G G^{*}, G^{*-1} P G^{*}\right) .
$$

Given $(A, E) \in \mathcal{Z}^{\circ}$, the orbit of $(A, E)$ given by the action $L$ is the set

$$
\mathcal{O}_{(A, E)}=\left\{(B, R) \in \mathcal{Z}^{\circ}:(B, R)=L_{G}((A, Q)) \text { for } G \in G L(\mathcal{H})\right\} .
$$

Observe that $\mathcal{O}_{(A, E)}=\mathcal{O}_{\left(I, E_{0}\right)}$, where $E_{0}=A^{1 / 2} E A^{-1 / 2} \in \mathcal{P}$. If $\mathcal{U}(\mathcal{H})$ denotes the subgroup of $G L(\mathcal{H})$ of unitary operators, then:

Proposition 6.9. Given $(A, E) \in \mathcal{Z}^{\circ}$ and $P \in \mathcal{P}$, then $(A, E) \in \mathcal{O}_{(I, P)}$ if and only if $E_{0}:=A^{1 / 2} E A^{-1 / 2} \in \mathcal{U}_{P}:=\left\{U P U^{*}: U \in \mathcal{U}(\mathcal{H})\right\}$.

Proof. Given $(A, E) \in \mathcal{Z}^{\circ}$ and $P \in \mathcal{P}$, suppose that $(A, E) \in \mathcal{O}_{(I, P)}$; then, there exists $G \in G L(\mathcal{H})$ such that $A=G G^{*}$ and $E=G^{*-1} P G^{*}$; or, equivalently, $A^{1 / 2}=\left|G^{*}\right|$, so that $G=A^{1 / 2} U$, with $U \in \mathcal{U}(\mathcal{H})$. Then, $E=A^{-1 / 2} U P U^{*} A^{1 / 2}$, or, $E_{0}=A^{1 / 2} E A^{-1 / 2}=U P U^{*}$. Therefore, $E_{0} \in \mathcal{U}_{P}$.

Conversely, if $E_{0} \in \mathcal{U}_{P}$ then there exists $U \in \mathcal{U}(\mathcal{H})$ such that $E_{0}=U P U^{*}$, or equivalently, $A^{1 / 2} E A^{-1 / 2}=U P U^{*}$, so that $E=A^{-1 / 2} U P U^{*} A^{1 / 2}$. Taking $G=A^{1 / 2} U$, then $G G^{*}=A$ and $E=G^{*-1} P G^{*}$. Hence, $(A, E) \in \mathcal{O}_{(I, P)}$.

Corollary 6.10. Given $(A, E) \in \mathcal{Z}^{\circ}$ and $P \in \mathcal{P}$, then $(A, E) \in \mathcal{O}_{(I, P)}$ if and only if $\operatorname{dim} \mathcal{R}(E)=\operatorname{dim} \mathcal{R}(P)$ and $\operatorname{dim} \mathcal{N}(E)=\operatorname{dim} \mathcal{N}(P)$, or equivalently if $E \in \mathcal{O}_{P}:=\left\{G P G^{-1}: G \in G L(\mathcal{H})\right\}$.

Proposition 6.11. The action $L$ is locally transitive and the map $\pi$ admits local cross sections.

Proof. Let us see that the action $L$ is locally transitive: given $\left(A_{0}, E_{0}\right) \in \mathcal{Z}^{\circ}$, we have to find an neighbourhood $V=V_{\left(A_{0}, E_{0}\right)}$ such that if $(A, E) \in V$ then there exists $G \in G L(\mathcal{H})$ such that $L\left(G,\left(A_{0}, E_{0}\right)\right)=(A, E)$. Suppose that such $G$ exists. Then $G$ verifies that

$$
G A_{0} G^{*}=A \text {, and } G^{*-1} E_{0} G^{*}=E .
$$

Therefore, $\left|A_{0}^{1 / 2} G^{*}\right|=A^{1 / 2}$ so that $G A_{0}^{1 / 2}=A^{1 / 2} U$, with $U$ unitary. Hence, $G=A^{1 / 2} U A_{0}^{-1 / 2}$.

Then, if $G^{*-1} E_{0} G^{*}=E$, it follows that $U A_{0}^{1 / 2} E_{0} A_{0}^{-1 / 2} U^{*}=A^{1 / 2} E A^{-1 / 2}$. Observe that the projections $P_{0}=A_{0}^{1 / 2} E_{0} A_{0}^{-1 / 2}$ and $P=A^{1 / 2} E A^{-1 / 2}$ are selfadjoint because $\left(A_{0}, E_{0}\right),(A, E) \in \mathcal{Z}^{\circ}$.

If the pairs $\left(A_{0}, E_{0}\right),(A, E)$ are close enough, the projections $P$ and $P_{0}$ verify that $\left\|P-P_{0}\right\|<1$, because of the continuity of the functions involved and the fact that $E_{0}$ and $E$ can be taken as close as necessary. Therefore it is possible to find an unitary operator $U$ such that $P=U P_{0} U^{*}$ : in fact $C=I-\left(P-P_{0}\right)^{2}$ 
provides a positive invertible operator such that the operator $U=P C^{-1 / 2} P_{0}+$ $(I-P) C^{-1 / 2}\left(I-P_{0}\right)$ is unitary and and $U P_{0} U^{*}=P$, see [14].

Using that $L$ is locally transitive it is easy to see that $\pi$ has local cross sections: in fact, if $s: U \longrightarrow G L(\mathcal{H}), s((A, E))=A\left(E A^{-1 / 2} C^{-1 / 2} P+(I-\right.$ E) $\left.A^{-1 / 2} C^{-1 / 2}(I-P)\right)$, with $C=I-\left(A^{1 / 2} E A^{-1 / 2}-P\right)^{2}$ and $U$ is a neighbourhood of $P$, given as before, then $\pi(s((A, E))=(A, E)$, for all $(A, E) \in U$. To obtain a local cross section for another point of $\mathcal{Z}^{\circ}$ consider $\left(A_{0}, E_{0}\right) \in \mathcal{O}_{(I, P)}$. Then, there exists $G \in G L(\mathcal{H})$ such that $L_{G}(I, P)=\left(A_{0}, E_{0}\right)$. Therefore, $\tilde{s}=l_{G} \circ s \circ L_{G^{-1}}$ is a local section in the neighborhood $G U$ of $\left(A_{0}, E_{0}\right)$.

Proposition 6.12. Let $(A, E),\left(A^{\prime}, E^{\prime}\right) \in \mathcal{Z}$. Then $(A, E),\left(A^{\prime}, E^{\prime}\right)$ belong to the same arc component if and only if $E$ and $E^{\prime}$ belong to the same arc component.

Proof. Suppose that $E$ and $E^{\prime}$ belong to the same arc component. Then there exists a continuous curve of projections $\gamma:[0,1] \longrightarrow \mathcal{Q}$ such that $\gamma(0)=E$ and $\gamma(1)=E^{\prime}$. Consider the curve $\beta(t)=\gamma(t)^{*} \gamma(t)+\left(I-\gamma(t)^{*}\right)(I-\gamma(t))$. Then $\beta(t) \subseteq G L(\mathcal{H})^{+}$and $\beta(t) \gamma(t)=\gamma(t)^{*} \beta(t)$, for every $t \in[0,1]$. Therefore the curve $(\beta(t), \gamma(t))$ connects the pairs $(\beta(0), E)$ and $\left(\beta(1), E^{\prime}\right)$ in $\mathcal{Z}$. But the positive operators $\beta(0)$ and $A$ belong to $\mathcal{Z}^{E}$, which is a convex set. Then the segment $t \beta(0)+(1-t) A$, for $0 \leq t \leq 1$, of positive operators joining them is included in $\mathcal{Z}^{E}$. Therefore the curve $(t \beta(0)+(1-t) A, E)$ joins $(\beta(0), E)$ with $(A, E)$ in $\mathcal{Z}$. In a similar way, the point $\left(\beta(1), E^{\prime}\right)$ are $\left(A^{\prime}, E^{\prime}\right)$ are arc connected in $\mathcal{Z}$. This shows that $(A, E),\left(A^{\prime}, E^{\prime}\right)$ are arc connected in $\mathcal{Z}$.

The converse is straightforward.

Remark 6.13. Observe that, for every $(A, E) \in \mathcal{Z}^{\circ}$ the fibres $\mathcal{Z}_{A}, \mathcal{Z}^{E}$ are homogeneous spaces: in the first case the unitary group acts on $\mathcal{P}$ by similarity and, in the second, the product group $G L\left(\mathcal{R}\left(E^{*}\right)\right) \times G L\left(\mathcal{N}\left(E^{*}\right)\right)$ acts on $\mathcal{Z}^{E}$ by restriction of the map $L$ defined above. See [3] for details on the geometric structure of positive operators on a Hilbert space. See also [9].

Acknowledgement. This research partially supported by FONCYT (PICT 808/08), UBACYT I023.

\section{REFERENCES}

1. T. Ando, Generalized Schur complements, Linear Algebra Appl. 27(1979) 173-186.

2. G. Corach, C. Gonzalez and A. Maestripieri, Unbounded symmetrizable idempotents, Linear Algebra Appl. 437 (2012), 659-674.

3. G. Corach and A. Maestripieri, Differential and metrical structure of positive operators, Positivity 4 (1999) 297-315.

4. G. Corach and A. Maestripieri, Products of orthogonal projections and polar decompositions, Linear Algebra Appl. 434 (2011), 1594-1609.

5. G. Corach, A. Maestripieri and D. Stojanoff, Oblique projections and Schur complements, Acta Sci. Math. (Szeged), 67 (2001) 337-356.

6. G. Corach, A. Maestripieri and D. Stojanoff, Generalized Schur complements and oblique projections. Linear Algebra Appl. 341 (2002), 259-272.

7. G. Corach, A. Maestripieri and D. Stojanoff, Oblique projections and abstract splines, J. Approx. Theory 117 (2002), 189-206. 
8. G. Corach, A. Maestripieri and D. Stojanoff, A classification of projectors, Banach Center Publications 67 (2005), 145-160.

9. M.E. Di Iorio Lucero, Geometry of the left action of the p-Schatten groups, Bannach J. Math. Anal. 7 (2013), 73-87.

10. T.N.E. Greville, Solutions of the matrix equation $X A X=X$, and relations between oblique and orthogonal projectors, SIAM J. Appl. Math. 26 (1974), 828-832.

11. P.R. Halmos, Introduction to Hilbert spaces and the theory of spectral multiplicity, Chelsea Publishing Company, New York, 1972.

12. S. Hass and K. Nordström, On projections in a space with an indefinite metric, Linear Algebra Appl. 208/209 (1994), 401-417.

13. M. Hestenes and E. Stiefel, Methods of conjugate gradients for solving linear systems. J. Research Nat. Bur. Standards 49 (1952), 409-436.

14. Y. Kato, An elementary proof of Sz-Nagy's theorem, Math. Japon.20 (1975), 257-258.

15. N. Kuiper, The homotopy type of the unitary group of Hilbert space, Topology 3 (1965), 1930.

16. A. Maestripieri and F. Martnez Pera, Decomposition of selfadjoint projections in Krein spaces Acta Sci. Math. (Szeged),72 (2006), 611-638.

17. P. Massey and D. Stojanoff, Generalized Schur complements and P -complementable operators, Linear Algebra Appl. 393 (2004), 299-318.

18. D. Pappas, Minimization of constrained quadratic forms in Hilbert spaces, Ann. Funct. Anal. 2 (2011), 1-12.

19. Z. Pasternak-Winiarski, On the dependence of the orthogonal projector on deformations of the scalar product. Studia Math. 128 (1998), 1-17.

20. R. Penrose, A generalized inverse for matrices, Proc. Cambridge Philos. Soc. 51 (1955), 406-413.

21. A. Sard, Approximation and variance. Trans. Amer. Math. Soc. 73 (1952), 428-446.

Departamento de Matemática, Facultad de Ingeniería,UBA, Buenos Aires(C1063ACV), Argentina;

Instituto Argentino de Matemática - COniCEt, SaAvedra 15, Buenos Aires (1083), Argentina.

E-mail address: gcorach@fi.uba.ar

E-mail address: amaestri@fi.uba.ar 